\title{
DETERMINATION OF EPILEPTIC DISORDER WITH DISCRETE WAVELET TRANSFORMS AND NEURAL NETWORK CLASSIFIER
}

\begin{abstract}
${ }^{1}$ S.T. Sadish Kumar and ${ }^{2}$ N. Kasthuri
${ }^{1}$ Electronics and Communication Engineering, Nandha Engineering College, Erode, Tamilnadu, India, ${ }^{2}$ Electronics and Communication Engineering, Kongu Engineering College, Perundurai, Erode, Tamilnadu, India

Received 2013-06-03; Revised 2013-08-01; Accepted 2013-11-12

ABSTRACT

Nowadays Epileptic disorder is a most challenge aspects in brain activation. Electroencephalograph (EEG) is one of the popular procedures to understand the human brain condition. The activation of brain will be changed due to the symptoms of neurological disorder. We have been proposed a procedure to find epilepsy disorder, using discrete wavelet transform and neural network classifier. The EEG classification has also been done by back propagation algorithm in DWT. Through back propagation algorithm in wavelet transform the EEG signal divided into sub bands. We have used low pass and high pass filters to scale and wavelet transformation of signals in order to perform filtering operation. Then the seizure will determined from sub bands. This study also discusses epilepsy disorder detection technique using neural network classifier with great accuracy.
\end{abstract}

Keywords: Electroencephalograph (EEG), Discrete Wavelet Transforms (DWT)

\section{INTRODUCTION}

Electrical activity of brain is identified by EEG technique. The voltage range of EEG signal is $1 / 100$ of voltage range of ECG signal. The particular value of voltage in EEG signal is 5-60 Hz. We can understand the symptoms of epileptic as group not as a single. This is the major symptoms neurological disorders in brain. The doctors will take more time to analyze the disorder. EEG is useful in the process of Epilepsy diagnosis, which reduces the time required and we have developed a framework using which Epilepsy of human brain could be detected.

The application of seizure detection is that by using this technique we can identify the brain activation. Moreover, by affording patients with other surgical way of treatments has produced some early detection of epilepsy or seizure prediction but takes more time of observation. In theory of early detection, the ictal manifestation in EEG is expected to be detected several seconds to a few minutes earlier.

The seizure detection is useful for identify more problems quickly, which is need to be know about destination and handled seriously to upcoming process. Several procedures are able to utilize the single feature separation, which is utilized by the previous saturative for decision making process. The noise will be occur in EEG signal, which is lead to find the abnormal thing that is find wrong identification in nonlinear method. The usage of nonlinear has been analyzed oven linear methods. Although the various studies method is very complicated, the selection of dataset will also be complex.

The clear statistical methods contain remains another major concern. Combine the two epileptic seizure detection methods are determined by the combination of logic gates "AND" and "OR". The result will be in highest sensitivity due to the combination of logic gates, the performance will also be improved. In this study,

Corresponding Author: S.T. Sadish Kumar, Electronics and Communication Engineering, Nandha Engineering College, Erode, Tamilnadu, India, 
four methods are used to combine the gate to find out the epileptic seizure early. The demonstration ensures that this approach will be a solid solution to mention.

\subsection{Related Work}

The input signal splitted into different frequency bands with various frequencies such as alpha, beta, theta and delta at the decomposition stage. This operation is performed using high and low pass filtering of signals.. Initially the input signal $i_{[n]}$ is given to half high and low pass filters namely $h, 1$. After the filtered process, sampling will be eliminated based on Nyquist's/2 radians due to the high frequency of signal. The signal will be sub sampled again by 2 , simplified by discarding other sample. The first level of decomposition can be expressed as follows:

$$
\begin{aligned}
\operatorname{high}[\mathrm{k}] & =\sum \mathrm{i}[\mathrm{n}] \cdot \mathrm{h}[2 \mathrm{k}-\mathrm{n}] \\
\operatorname{low}[\mathrm{k}] & =\sum \mathrm{i}[\mathrm{n}] \cdot 1[2 \mathrm{~h}-\mathrm{n}]
\end{aligned}
$$

Here high[k] represent the output produced by high pass filter where as low $[\mathrm{k}]$ shows the output produced by low pass filter after sub sampling by 2 . We can reduce the overall time required by $50 \%$ using decomposition method. The entire signal is characterized by the $1 / 2$ part of the number of samples. The frequency band will carry the previous band only, despite of increase the frequency resolution. The above sub band coding operation is used for decompose the signal in further.

Sukanesh and Harikumar (2012) explain discusses how the information theory could be adapted for optimization of fuzzy outputs in risk level epilepsy classification. They have compared two methods with their performance indices and quality values. Pan et al. (2009) said that an approach called locally linear embedding and Weighted Locally Linear Embedding have been developed for internal feature extraction. Locally linear embedding an unsupervised learning method which computes low-dimensional neighborhood but preserves the embedding of high-dimensional inputs. The LLE differs from clustering methods in local dimensionality reduction i.e., LLE maps its inputs into a single global coordinate system of lower dimensionality and its optimizations will not participate in local minima. LLE is able to learn the global structure of data set by exploiting the local symmetries.

Johnson et al. (2011) discusses the generative and discriminative approaches to classification of epileptic seizure. The generative method compares the model with training data for each category based on likelihood or mean square error. It classifies the EEG based on the score generated for each model and selects the model which has more optimum score whereas the discriminative approach directly estimate the boundaries between different classes.

Streams applications are represented as directed data flow graphs consisting of a group of elements, which are connected by data streams. Each group of data stream carries a series of stream data elements. At the operating system level, PEs lives in their own processes and communicates with each other via their input and output ports, using the TCP/IP network stack. Input and output ports are connected by streams to form these directed data flow graphs.

The deconvolution of the speech signal to remove the noise is applicable to removing the inherent environmental noise associated with recording EEG. As in speech recognition, neural changes in EEG are dominant in the frequency domain. Supervised learning algorithms have been applied to this feature set to classify windows of EEG data. The Multi-channel Epileptic (MCE) efficiency is due to its ability to make use of lightweight classifier for maximum efficiency. This is especially appealing in the context of real-time seizure detection, in which a smaller, less complex, but highly efficient classifier is adapted more too lightweight devices used by medical staffs for patient care. This result is in line with the MCE philosophy as it emphasizes all classes equally, weighting them for minimum error purposes.

Nasehi and Performed (2011) a survey of various seizure detection algorithms and their application in various fields. Most recent algorithms use EEG and ECG signals to detect the seizure. They identify three seizure detectors; EEG-based seizure-event detectors, EEG based seizure-onset detectors and EEG/ECG-based seizure-onset detectors.

Omerhodzic et al. (2010) proposed a Wavelet-based Neural Network (WNN) classifier for recognizing EEG signals is implemented and tested under three sets EEG signals (healthy subjects, patients with epilepsy and patients with epileptic syndrome during the seizure). First, the Discrete Wavelet Transform (DWT) with the Multi-Resolution Analysis (MRA) is applied to decompose EEG signal at resolution levels of the components of the EEG signal (delta, theta, alpha, beta and gamma) and the Parsevals theorem are employed to extract the percentage distribution of energy features of the EEG signal at different resolution levels. Second, the Neural Network (NN) classifies these extracted features to identify the EEGs type according to the percentage distribution of energy features.

Guo et al. (2010a) presents a novel method for automatic epileptic seizure detection, which uses approximate entropy features. Multi wavelet transform is 
used to generate the features and it uses artificial intelligence neural network for classification.

Guo et al. (2010b) discusses an approach using line length features extracted using wavelet transform multi resolution decomposition and uses ANN for the classification of EEG signals.

Filligoi et al. (2011) describe a software tool to analyze the long term Holter-EEG and a template matching algorithm which uses statistical approach to compare the EEG with the EEG of known epileptics data base.

Cherian et al. (2011) proposed a classification and validation system using EEG background classification. It uses eight classes to classify the EEG signals using their discontinuity, sleep and wake up cycle.

Manimegalai (2012) presents wavelet decomposition based EEG classification method. Delta signal is detected by multilevel decomposition and neural network is used to classify the extracted delta signal.

Raghunathan et al. (2010) presents a twodimensional design optimization approach that considers detection efficiency and hardware cost in evaluating algorithms. Detection features are evaluated for electrographic seizure detection ability from microelectrode data recorded in kainite treated rats. Dynamic and leakage power consumption is estimated using circuit models. Raghunathan et al. $(2010 ; 2011)$ present a two-dimensional design optimization approach that takes into account both detection efficacy and hardware cost in evaluating algorithms for their feasibility in an implantable application. Detection features are first compared for their ability to detect electrographic seizures from micro-electrode data recorded from kainate-treated rats. Circuit models are then used to estimate the dynamic and leakage power consumption of the compared features. A score is assigned based on detection efficacy and the hardware cost for each of the features, then plotted on a two-dimensional design space.

Sharanreddy and Kulkarni (2011) present a review of the significant researches associated with the automated detection of epileptic seizures and brain tumor using EEG signals.

Mercy (2012) conducted the performance analysis of automatic detection of EEG patterns using discrete wavelet Transform and Independent Component Analysis. For feature extraction DWT and ICA methods are used and svm and neural network is used for classification.

Chandler et al. (2011) presents a low power platform which performs continuous multi-channel detection and analysis of seizures for epilepsy patients. On detection of seizure the detection unit triggers the analysis circuit which locally processes the signal and sends the energy and frequency content.

Majumdar (2011) show that differentiation can enhance certain features of brain electrophysiological signals, contaminated with noise, artifacts and acquisition defects, leading to efficient detection of those changes. They used windowed variance method for the detection of seizure EEG signals. They used both windowed variance and automatic seizure detection to provide real time support for epileptic seizure detection in order to reduce the processing time.

Lewis et al. (2010) presents a system that combines power spectra of seizures deterministic finite automata. They have used these combinations for the prediction and detection of epileptic from activity.

Bisasky et al. (2012) presents a reconfigurable manycore platform performing fixed point DSP applications supporting up to 64 cores routed in a hierarchical network. The individual cores are based on a 5 stage RISC pipeline architecture optimized to support communication to other cores on the platform. Communication between cores is implemented using low-area routers that partitions computational cores into hierarchical clusters resulting in a low network diameter. The routers use a packet-switched protocol that minimizes circuitry which further reduces circuit size in comparison to the computational circuitry.

\section{MATERIALS AND METHODS}

The EEG signals gathered from different patients. This signal is transferred to another format. The process is done using MATLAB simulation tool. The Fig. 1 shows the overall architecture of the epileptic seizure detection. It has various functional models namely preprocessing, band separation, scaling functions to filter the signals, neural network classifier and finally epileptic seizure detection. The proposed method takes the EEG signal as the input and the formatted signal is given to the pre-processing operation block. The pre-processing operation is to reduce the unwanted noisy signals from input signal which intrude at the time of EEG recording. The next block to pre-processing is Discrete Wavelet Transform, in which the input signal is divided into four sub bands with different frequency. This process is called as decomposing. Out of these sub bands, the seizure is detected from delta band. The Neural network classifier works with Discrete Wavelet Transform to train the Delta wave. The Back Propagation Algorithm (BPA) compares the waves with normal condition and with epileptic using neural network classifier. 


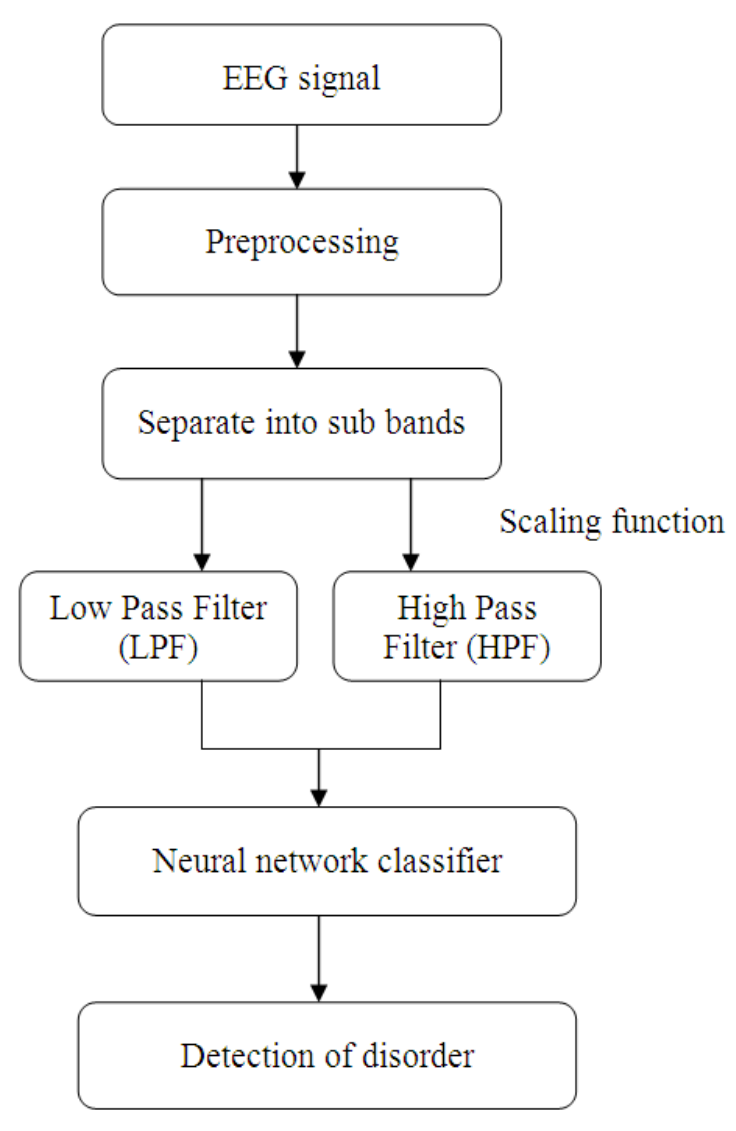

Fig. 1. Epileptic disorder detection

Finally the seizure will be determined from the output of Neural Network Classifier. We have been record the EEG signal up to $100 \mathrm{~Hz}$ from various patients. But up to $60 \mathrm{~Hz}$ only used for analyzing the epileptic detection using Root Mean Square (RMS) software. So we have to use band pass filter to trip the signal frequency up to $60 \mathrm{~Hz}$.

The unwanted noise should be eliminated before processing the EEG signal. The high pass filter is used to reduce the noise up to $50 \mathrm{~Hz}$. In next step, the signal is decomposed into different sub bands with various frequency i.e., alpha $(9-13 \mathrm{~Hz})$, beta (14-30 $\mathrm{Hz}$ ), theta (4-8 Hz), delta (1-3 Hz), using DWT. DWT contains two sets of functions, which are named as scaling functions for low pass filter and wavelet functions for high pass filter.

\subsection{Architecture of Back Propagation Network}

The generalized form of least mean squared algorithm is called the BPA which modifies the network weights in order to reduce the mean squared error between desired and actual output of the network. The supervised learning is used to train the data where the desired and actual outputs are known. The trained data are used to compute network weights based on which new input samples are computed. The forward method is used to provide input pattern to the input layer neurons that pass values into hidden layer. Every hidden layer performs the same task to pass the results to the output layer.

The nodes at the input layer transmit input values to the nodes at hidden layer without perform any computation. The dimensionality of input pattern is equivalent to the number of input nodes. The number of nodes at the output layer is depending on the problem considered. For example the problem is to map ndimensional input vectors into m-dimensional output vectors, then the network contains $\mathrm{n}$ input nodes and $\mathrm{m}$ output nodes. To treat bias or threshold as other weights in the network a dummy input node with constant input is used. The number of hidden layer nodes is up to the network designer and depends on problem complexity. The nodes present other than input layer uses a sigmoid function to its net input.

\subsection{Training Algorithm}

The procedure of training algorithm is presented as follows.

Let $\mathrm{X}$ is the input training vector and $\mathrm{t}$ be the target vector:

$X=(x 1, x 2 \ldots x n)$

$\mathrm{T}=(\mathrm{t} 1, \mathrm{t} 2 \ldots \mathrm{tm})$

$\delta \mathrm{k}=$ error at output unit $\mathrm{Y}_{\mathrm{k}}$

$\delta \mathrm{j}=$ error at hidden unit $\mathrm{Zj}$

Let $\alpha$ is the learning rate and $v_{o j}$ is the bias on hidden unit $\mathrm{j}$ and $\mathrm{Z}_{\mathrm{j}}$ is the output on hidden unit $\mathrm{j}$. The bias on output unit $\mathrm{k}, \mathrm{Y}_{\mathrm{k}}$ is the output unit $\mathrm{k}$ :

Step 1: Read EEG signal and record that, from different patient.

Step 2: $\quad$ Reduce the noise from EEG signal by using DWT.

Step 3: Apply low pass and high pass filtering using scaling and wavelet function.

Step 4: Apply the BPA in neural network classifier for each input unit.

Step 5: The Hidden Unit (HU) is considered as $\mathrm{Zj}$, where $\mathrm{j}$ varies from 1 to $\mathrm{p}$ and accumulate its weighted input signals:

$$
\mathrm{Z}_{\mathrm{inj}}=\mathrm{V}_{\mathrm{oj}}+\sum \mathrm{x}_{\mathrm{i}} \mathrm{v}_{\mathrm{ij}}
$$


Step 6: Apply activation function, $\mathrm{Zj}=\mathrm{f}$ (Zinj) and send to layer above.

Step 7: Each output unit $\mathrm{Y}_{\mathrm{k}}, \mathrm{k}=1$ to $\mathrm{m}$ sums its weighted input signals:

$$
\mathrm{Y}_{\mathrm{ink}}=\mathrm{W}_{\mathrm{ok}}+\sum \mathrm{z}_{\mathrm{j} \mathrm{j} \mathrm{k}}
$$

Apply its activation function to calculate the output signal $\mathrm{Y}_{\mathrm{k}}=\mathrm{f}\left(\mathrm{Y}_{\mathrm{ink}}\right)$.

Step 8: Separate into sub bands the input signal with various frequency.

Step 9: at each hidden unit $\mathrm{Zj}$, where $\mathrm{j}=1$ to $\mathrm{m}$ sums its $\delta$ inputs from the units in the layer above:

$$
\operatorname{Sinj}=\sum_{\mathrm{k}=1}^{\mathrm{m}} \delta \mathrm{jWjK}
$$

The error information term is calculated as $\Delta \mathrm{j}$ $=$ Sinj f' (Zinj)

Step 10: Update weights and biases at each unit of output $\mathrm{Y}_{\mathrm{K}}$, with $\mathrm{k}=1$ to $\mathrm{m}$. The weight correction term is given by:

$$
\begin{aligned}
& \Delta \mathrm{WjK}=\delta \mathrm{kZj} \\
& \Delta \mathrm{WoK}=\alpha \delta \mathrm{k}
\end{aligned}
$$

Thus:

$$
\begin{aligned}
\mathrm{WjK}(\text { new }) & =\mathrm{WjK}(\text { old })+\Delta \mathrm{WjK} \\
\mathrm{WoK}(\text { new }) & =\mathrm{WoK}(\text { old })+\Delta \mathrm{WoK}
\end{aligned}
$$

Each hidden unit $\mathrm{Zj}, \mathrm{j}=1$ to $\mathrm{p}$, update its weight and bias. The weight correction term is given by:

$$
\begin{gathered}
\Delta \mathrm{Vij}=\delta \Delta \mathrm{j} \mathrm{Xj} \\
\Delta \operatorname{Voj}=\delta \Delta \mathrm{j}
\end{gathered}
$$

Thus:

$$
\begin{aligned}
\operatorname{Vij}(\text { new }) & =\operatorname{Vij}(\text { old })+\Delta \operatorname{Vij} \\
\operatorname{Voj}(\text { new }) & =\operatorname{Voj}(\text { old })+\Delta \operatorname{Voj}
\end{aligned}
$$

Step 11: Detect the Epileptic disorder from delta signal.

\subsection{Parameter selection for BPA}

In order to choose initial weight for best results initial weights has been set in the range of -0.5 to 0.5 or -1 to 1 . The learning rate $\alpha$ : With higher value leads to rapid learning but the weights may oscillate and lower value slower the learning process. Proposed method to choose learning rate is as follows:

- Initialize learning rate with higher value and steadily decrease it. To reduce the oscillations reduce the weights at small range

- Increase the learning rate to worsen performance

- Back propagation learning

- Sequential learning or pre-pattern method

- Batch learning or pre-epoch method

Sequential learning: The input pattern given at input layer is forward and the error rate is determined and propagated backwards and the weights are updated. In batch learning: The updating of weights is done only after the training of entire network.

In BPN the change of weight is at one direction effect with current gradient and previous one. The convergence will be faster if the momentum is added to weight. The weights must be saved at each training pattern to preserve the momentum. For BPN with momentum, the new weights for training step $(t+2)$ is based on $(t)$ and $(\mathrm{t}+1)$ and the net is allowed to do large weight adjustment if the direction is same for many patterns by momentum. The weight updating formula for BPN with momentum is given by:

$$
\begin{aligned}
\mathrm{W}_{\mathrm{j}} \mathrm{k}(\mathrm{t}+1) & =\mathrm{w}_{\mathrm{j}} \mathrm{k}(\mathrm{t})+\alpha \mathrm{kz}_{\mathrm{j}}+\mathrm{M}\left[\mathrm{w}_{\mathrm{j}} \mathrm{k}(\mathrm{t})-\mathrm{w}_{\mathrm{j}} \mathrm{k}(\mathrm{t}-1)\right] \\
\mathrm{V}_{\mathrm{j}} \mathrm{k}(\mathrm{t}+1) & =\mathrm{V}_{\mathrm{j}} \mathrm{k}(\mathrm{t})+\alpha \beta_{\mathrm{j}} \mathrm{x}_{\mathrm{j}}+\mathrm{M}\left[\mathrm{v}_{\mathrm{j}} \mathrm{k}(\mathrm{t})-\mathrm{v}_{\mathrm{j}} \mathrm{k}(\mathrm{t}-1)\right]
\end{aligned}
$$

In this approach the patient EEG does not match with input $70 \%$ after certain number of iterations then the patient is diagnosed as having epileptic.

\section{RESULTS AND DISCUSSION}

Epileptic seizure detection in EEG records is a useful and important tool due to their various applications such as epilepsy research treatments like timely drug delivery, electrical stimulation and seizure alert systems besides diagnostic applications. In this sense it is a real need the development of automatic algorithms that could be able to detect seizures independently of its brain source. It is also important to establish some kind of standardization of the detectors using to test them the same database so a robust comparison of their performance could be carried out.

The Table 1 shows the efficiency and accuracy of epileptic detection by various methods. It shows clearly that the proposed DWT with Neural network has higher detection rate and takes lesser time than other methods. 
Table 1. Comparison between various methods and DWT

\begin{tabular}{lll}
\hline Epilepsy detection & $\begin{array}{l}\text { Detection } \\
\text { rate }\end{array}$ & $\begin{array}{l}\text { Time } \\
(\mathrm{sec})\end{array}$ \\
\hline $\begin{array}{l}\text { Support Vector Machine (SVM) } \\
\begin{array}{l}\text { Minimum relative entropy with } \\
\text { fuzzy logic }\end{array}\end{array}$ & $80 \%$ & 10 \\
$\begin{array}{l}\text { Fisher's discriminant analysis } \\
\text { with linear classifier }\end{array}$ & $90 \%$ & 8 \\
\begin{tabular}{l} 
DWT and neural network \\
\hline
\end{tabular} & $98 \%$ & 7 \\
\hline
\end{tabular}

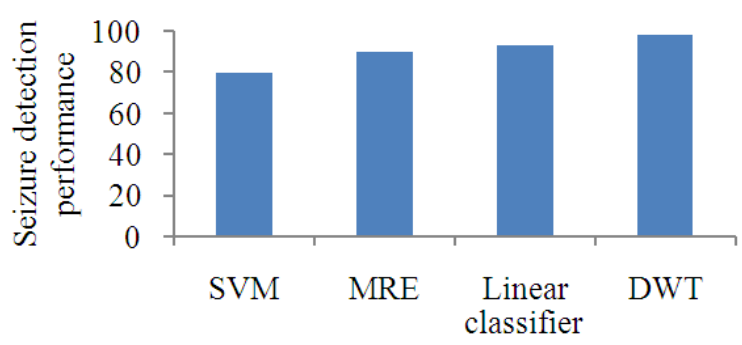

Methods for seizure detection

Fig. 2. Graphical representation of seizure detection rate performance

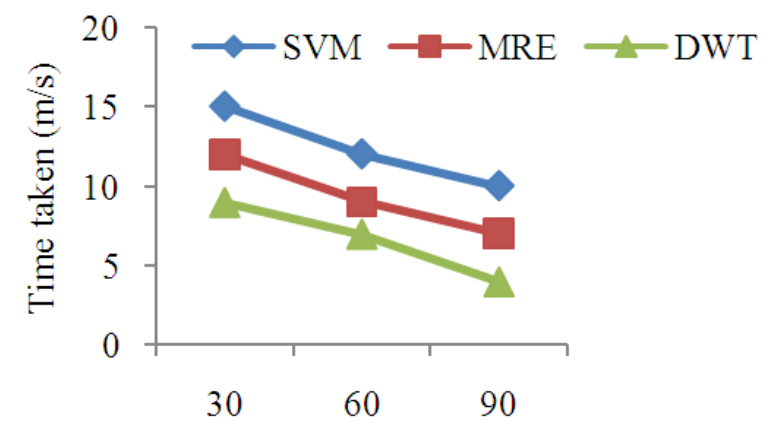

Accuracy of various methods for seizure detection

Fig. 3. Graphical representation of processing time performance

In this chapter two epileptic seizure detection methods based on the Discrete Wavelet Transform (DWT) of EEG signals has been proposed. In addition, as a contribution to the setted out problem, long term epileptic EEG intracranial records with different focal epilepsies are used to evaluate the performance of both seizures detectors.

In Fig. 2, the performance of discrete wavelet transform is shown, which is compared with other methods based on accuracy. The Discrete Wavelet Transform is having more accuracy than other techniques.
In Fig. 3 the processing of time performance has been shown below. In DWT with neural network method, the classification would be done in $4 \mathrm{sec}$ and the accuracy increases up to more level.

\section{CONCLUSION}

Form discussion, we can use Discrete Wavelet Transform for EEG signal analysis for great accuracy. In previous approach we can identify the seizure in brain activation with the lowest accuracy. Also the performance of those methods has been taken more time to detect the seizure. This study proposes a novel method to detect epilepsy disorder using discrete wavelet transform which implemented in MATLAB. The network is trained in neural network and for the classification we have used Back Propagation Algorithm (BPA). The experimental results show that the epilepsy detection could be done within short time of seconds using the proposed methodology. The proposed method produces the epileptic detection accuracy up to $98 \%$.

\section{REFERENCES}

Bisasky, J., D. Chandler and T. Mohsenin, 2012. A many-core platform implemented for multi-channel seizure detection. Proceedings of the IEEE International Symposium on Circuits and Systems, May 20-23, IEEE Xplore Press, Seoul, pp: 564-567. DOI: 10.1109/ISCAS.2012.6272092

Chandler, D., J. Bisasky, Stanislaus, J.L.V.M. and T. Mohsenin, 2011. Real-time multi-channel seizure detection and analysis hardware. Proceedings of the IEEE Biomedical Circuits and Systems Conference, Nov. 10-12, IEEE Xplore Press, San Diego, CA., pp: 41-44. DOI: 10.1109/BioCAS.2011.6107722

Cherian, P.J., W. Deburchgraeve, R.M. Swarte, M. De Vos and P. Govaert et al., 2011. Validation of a new automated neonatal seizure detection system: A clinician's perspective. Clin. Neurophysiol., 122: 1490-1499. PMID: 21396883

Filligoi, G., M. Padalino and S. Pioli, 2011. A matlab software for detection and counting of epileptic seizures in 72 hours Holter-EEG. Cyber J.

Guo, L., D. Rivero and A. Pazos, 2010a. Epileptic seizure detection using multiwavelet transform based approximate entropy and artificial neural networks. J. Neurosci. Methods, 193: 156-163. DOI: 10.1016/j.jneumeth.2010.08.030 
Guo, L., D. Rivero, J. Dorado, J.R. Rabunal and A. Pazos, 2010b. Automatic epileptic seizure detection in EEGs based on line length feature and artificial neural networks. J. Neurosci. Methods, 191: 101109. DOI: $10.1016 /$ j.jneumeth.2010.05.020

Johnson, A.N., B. Sow and A. Biem, 2011. A discriminative approach to EEG seizure detection. AMIA Annu. Symp. Proc., 2011: 1309-1317.

Lewis, R.A., B. Parks and A.M. White, 2010. Determination of epileptic seizure onset from EEG data using spectral analysis and discrete finite automata. Proceedings of the IEEE International Conference on Granular Computing, Aug. 14-16, IEEE Xplore Press, San Jose, CA., pp: 277-282. DOI: $10.1109 / \mathrm{GrC} .2010 .140$

Majumdar, K., 2011. Differential operator in seizure detection. Comput. Biol. Med., 42: 70-74. DOI: 10.1016/j.compbiomed.2011.10.010

Manimegalai, S.S., 2012. Detection of epilepsy disorder in EEG signal. Int. J. Emerg. Trends Eng. Dev., 2: 473-473.

Mercy, M.S., 2012. Performance analysis of epileptic seizure detection using DWT and ICA with neural networks. Int. J. Comput. Eng. Res., 2: 1109-1113.

Nasehi, S. and H. Pourghassem, 2011. A novel epileptic seizure detection algorithm based on analysis of EEG and ECG signals using probabilistic neural network. Aus. J. Basic Applied Sci., 5: 308-315.
Omerhodzic, I., S. Avdakovic, A. Nuhanovic and K. Dizdarevic, 2010. Energy distribution of EEG signals: EEG signal wavelet-neural network classifier. World Acad. Sci. Eng. Technol., 37: 1240-1245.

Pan, Y., S.S. Ge and A.A. Mamun, 2009. Weighted locally linear embedding for dimension reduction. Patt. Recogn., 42: 798-811. DOI: 10.1016/j.patcog.2008.08.024

Raghunathan, S., A. Jaitli and P.P. Irazoqui, 2011. Multistage seizure detection techniques optimized for low-power hardware platforms. Epilepsy Behav., 22: 61-68. DOI: 10.1016/j.yebeh.2011.09.008

Raghunathan, S., S.K. Gupta, H.S. Markandeya, K. Roy and P.P. Irazoqui, 2010. A hardware-algorithm codesign approach to optimize seizure detection algorithms for implantable applications. J. Neurosci. Methods, 1: 106-117. DOI: 10.1016/j.jneumeth.2010.08.008

Sharanreddy, M. and P.K. Kulkarni, 2011. Review of significant research on EEG based automated detection of epilepsy seizures and brain tumor. Int. J. Scientif. Eng. Res., 2: 1-9.

Sukanesh, R. and R. Harikumar, 2012. Minimum Relative Entropy (MRE) Method for fuzzy based classification of epilepsy risk levels from EEG signals. Int. J. Soft Comput. Eng. 